\title{
Data Driven Design Optimization Methodology Development and Application
}

\author{
H. Zhao, D. Knight, E. Taskinoglu, and V. Jovanovic \\ Dept of Mechanical and Aerospace Engineering \\ Rutgers - The State University of New Jersey \\ New Brunswick, NJ 08903 \\ knight@soemail .rutgers.edu
}

\begin{abstract}
The Data Driven Design Optimization Methodology (DDDOM) is a Dynamic Data Driven Application System (DDDAS) developed for engineering design optimization. The DDDOM synergizes experiment and simulation in a concurrent integrated software system to achieve better designs in a shorter time. The data obtained from experiment and simulation dynamically guide and redirect the design optimization process in real or near-real time, and therefore realize the full potential of the Dynamic Data Driven Applications Systems concept. This paper describes the DDDOM software system developed using the Perl and Perl/Tk programming languages. The paper also presents the first results of the application of the DDDOM to the multi-objective design optimization of a submerged subsonic inlet at zero angle of attack and sideslip.
\end{abstract}

\section{Introduction}

A Dynamic Data Driven Application System (DDDAS) is an application which can interact with experiment and/or simulation in real-time, building a bridge between them and guiding them dynamically [1. DDDAS is an important area of current research in Information Technology (IT) with a wide range of engineering and scientific applications [234]. Engineering design optimization can benefit significantly from the DDDAS concept whereby the concurrent integration of remote real-time input from high performance computing resources (e.g., NSF Teragrid 5) and experiments will achieve the best designs. D. Knight, G. Elliott, Y. Jaluria, N. Langrana and K. Rasheed proposed the Data Driven Design Optimization Methodology (DDDOM) [6] for Multi-criteria Design Optimization (MDO) 7 as a Dynamic Data Driven Application System. DDDOM therefore differs from the conventional, sequential engineering design approach. DDDOM utilizes advanced experimental technology (e.g., Rapid Prototyping) and computational technology (e.g., parallel processing, grid computing [5]). The DDDOM software system under development is shown in Fig. 1 It is comprised of six elements: User Interface, Controller, Optimizer, Experiment, Surrogate Model (SM) and Simulation. Further details are presented in Knight et al [8]. 


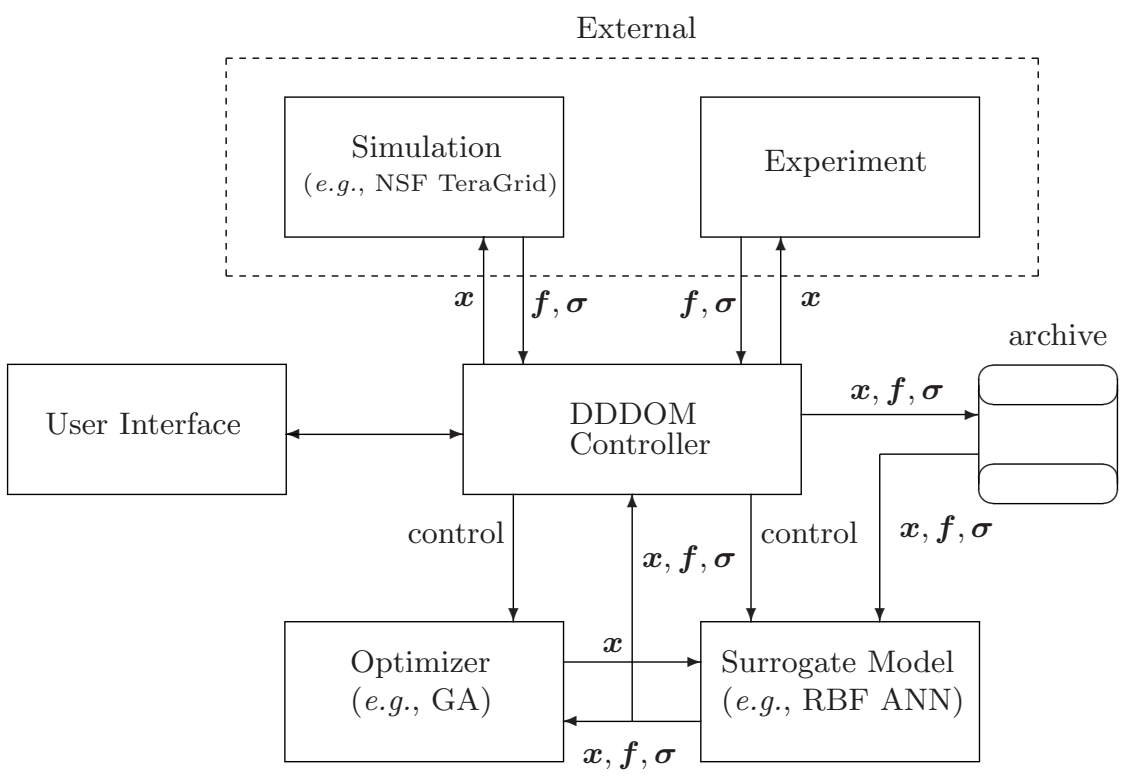

Fig. 1. Data Driven Design Optimization Methodology (DDDOM)

\section{The DDDOM Controller}

The DDDOM Controller is the central element in the DDDOM and controls the overall operations of the DDDOM cycle. Its basic function is to initiate and monitor the design optimization, and to direct the simulations and experiments needed to build and update the surrogate models. The functions are illustrated in Fig. 11and are described below.

Control of Optimizer: The Controller initiates the optimization and monitors the progress of the optimization. The Controller monitors each design, denoted by its vector of design variables $\boldsymbol{x}$, generated by the optimizer and the resultant vector of objective functions $\boldsymbol{f}$ and uncertainty $\boldsymbol{\sigma}$ generated by the Surrogate Model (SM). The Controller determines when new data is needed for training the SM and initiates new simulations and identifies new experiments needed.

Control of Surrogate Model: The Controller manages the state of the SM between training and modelling. It decides when the SM must be retrained using data stored on the archive.

Control of Simulation: The Controller determines the design $\boldsymbol{x}$ to be simulated and stores the resultant objective function $f$ and uncertainty $\boldsymbol{\sigma}$ on the archive.

Control of Experiments: The Controller determines the design $\boldsymbol{x}$ to be examined by experiment and requests the experiment to be performed. In certain cases, the experiment may be conducted automatically. 

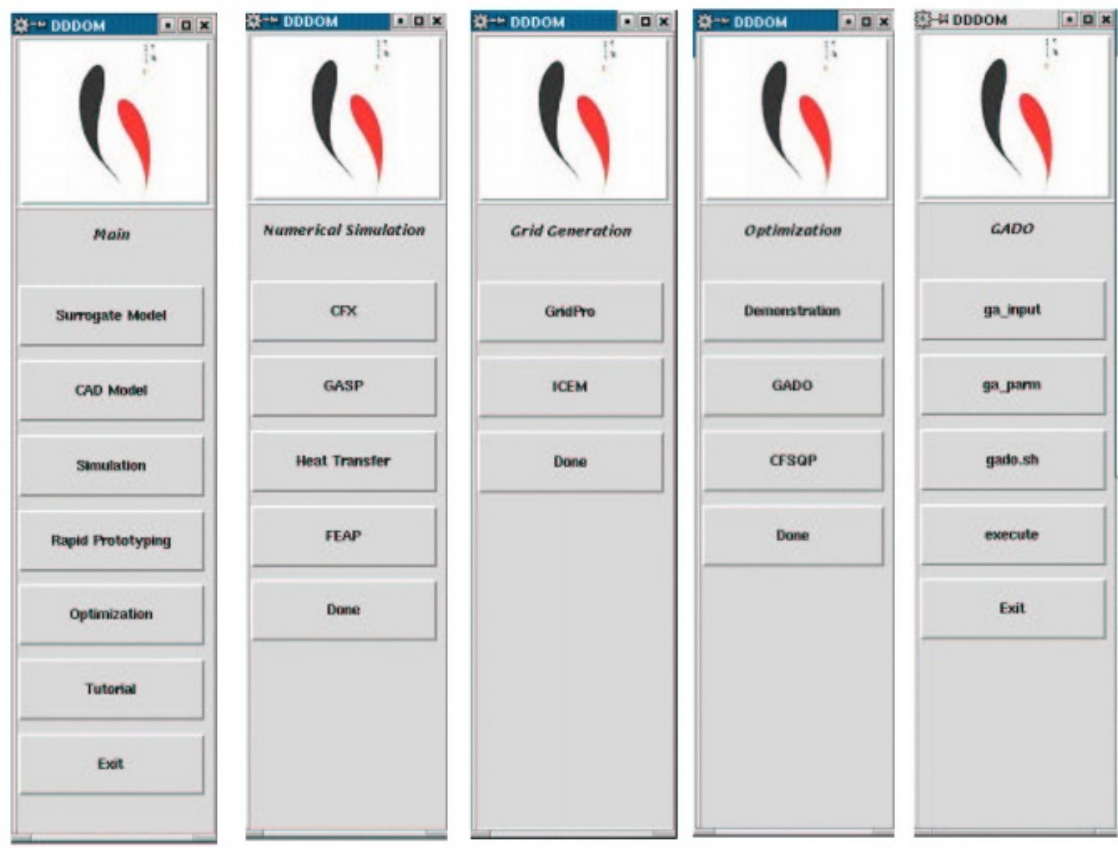

Fig. 2. Main panel for DDDOM controller

The DDDOM Controller must deal with executable codes, link different files and directories, and connect to databases. The User Interface is the access to the DDDOM Controller. The User Interface must be closely combined with the DDDOM Controller. It must be user-friendly, block-structured and well documented. The Controller is written in Perl and Perl/Tk 910[11. The main panel of the DDDOM User Interface is shown in Fig. 2

\section{Multi-objective Design Optimization}

\subsection{Problem Statement}

The DDDOM software system is applied to the multi-objective design optimization of a submerged inlet. A generic submerged inlet is shown in Fig. 3. Basically, it is a fluid flow duct through which air is delivered to a turbojet, turbofan or ramjet. The main objective for an inlet designer is to provide a high quality air flow, i.e., the optimum levels of total pressure and flow uniformity which are vital for engine operability and stability. Because of the curved shape, inertial forces create a swirl in the inlet leading to flow non-uniformity. The specific design objectives are to minimize the Distortion Coefficient (DC) and Swirl Coefficient 


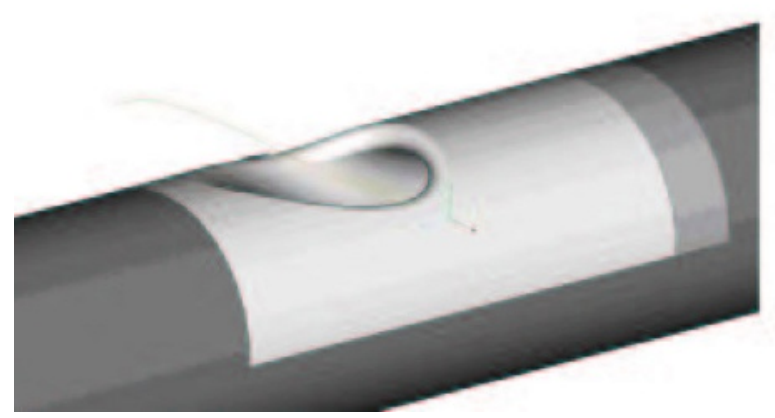

Fig. 3. Generic inlet

(SC) at the engine face (for definition, see [12]). Since the DC and SC are competing design objectives, the optimization problem is multi-objective and the solution is the Pareto Set of optimal designs.

This problem was selected to demonstrate the capability of the DDDOM to achieve an optimal set of designs (the Pareto Set) through concurrent integration of experiment and simulation while synergizing the respective advantages of each. For example, the computational cost of each simulation is independent of the inlet geometry, angle of attack or sideslip, and requires hundreds of total cpu hours. Since the simulation code is parallelized, the typical turnaround time for a simulation is six hours on the cluster at the Rutgers School of Engineering. The time to set up the experiment (including fabrication of the inlet model) is typically one day. However, changes in the inlet angle of attack or sideslip can be accomplished in a matter of minutes in the experiment. Thus, experiment and simulation can be synergized to achieve a more effective and efficient design optimization whereby the simulation is used to explore changes in the inlet geometry, and the experiment is used to explore changes in the inlet angle of attack and sideslip.

We present the results of the first stage of the multi-objective design optimization of a submerged inlet using experiment and simulation. In this stage, we conduct a design optimization of the inlet at zero angle of attack and sideslip using simulation alone, and then compare the predicted results for DC with the experiment. This establishes a baseline for calibration of the simulation against the experimental results which is an important element in the DDDOM. In the second stage (currently in progress), we will synergize the experiment and simulation in a concurrent manner to obtain the Pareto Set of designs for the case of non-zero angle of attack and sideslip.

Trade studies have shown 1213 that the inclusion of a fin inside the inlet provides a significant improvement in flow quality (Fig. 4). The design variables are therefore taken to be the length, width and angle of attack of the fin (Fig. 4(a)). 


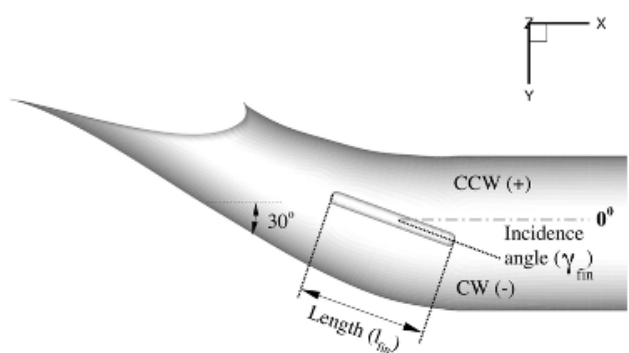

(a) Side view

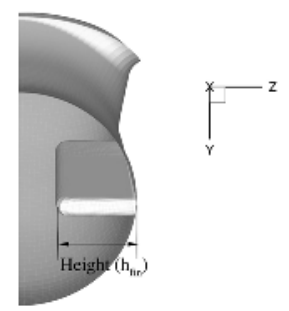

(b) View looking upstream

Fig. 4. Design variables for a fin type of deformation

\subsection{Design Optimization}

The design optimization incorporates five software components linked together in an automated loop built using Perl/Tk. The components are the Optimizer (CFSQP), Geometry Modeller (Pro/Engineer 14]), Mesh Generator (GridPro [15]), Flow Solver (GASPex [16]) and Auxiliary Program (to compute DC and SC). The Optimizer selects a specific geometry for the inlet. The geometry is passed to the Geometry Modeler (Pro/Engineer) which constructs a Computer Aided Design model of the inlet including the surface alteration in the form of a fin. The inlet surface definition is provided to the Mesh Generator (GridPro) which creates the grid in the flow domain. The grid is provided to the flow solver (GASPex) which solves the Reynolds-averaged compressible time dependent Navier Stokes equations in three dimensions. The computed flowfield is provided to the Auxiliary Program which calculates the objective functions DC and SC. The objective functions are provided to the Optimizer which then selects a new design. The loop continues, until the set of optimal designs (Pareto Set) is obtained. The entire process is controlled using Perl/Tk and the software components are executed on distributed systems based upon the licensing arrangements.

The set of designs examined in the optimization and the Pareto Set are shown in Fig. 15. The Pareto Set is disjoint and is characterized by two discrete sets of designs denoted A and B (circled). The designs in set A and set B show fundamentally different flow behavior. Set A designs are characterized by high swirl coefficient SC and low distortion coefficient DC, while Set B designs have low swirl coefficient SC and a higher distortion coefficient DC. Both sets of designs have improved values of both DC and SC compared to the baseline inlet without the fins $[12$.

\subsection{Experiment}

The experimental portion [12] of the inlet optimization problem is conducted in the subsonic wind tunnel located in the Low Speed Wind Tunnel Labora- 


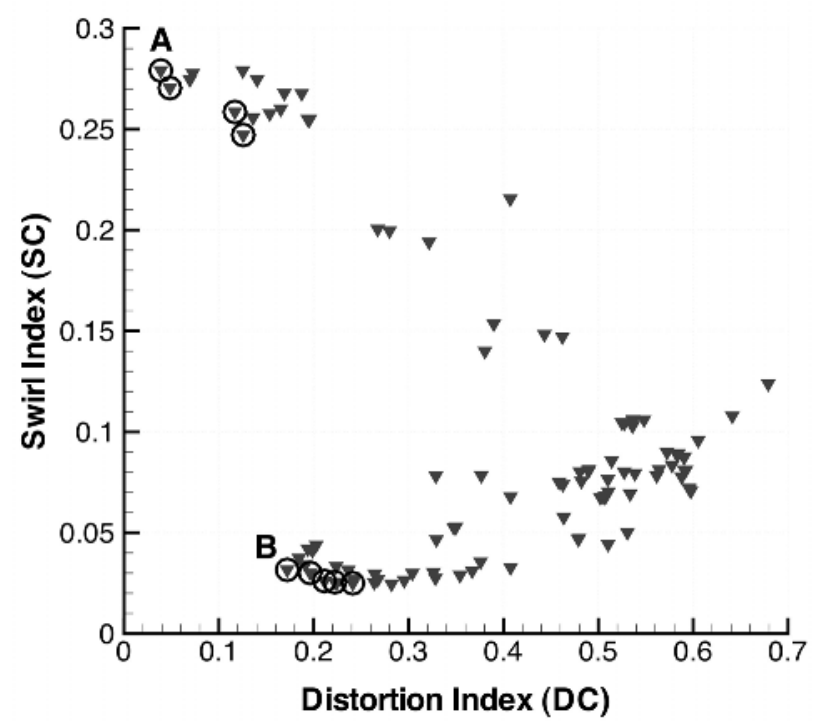

Fig. 5. Set of designs including Pareto Set (circled)

tory at Rutgers University (Fig. 6). A stainless steel model has been fabricated and installed into the test section of the subsonic wind tunnel. The model was designed with a removable submerged inlet (Fig. 7), which is fabricated using the Stratasys FDM Model 3000 rapid prototyping system at Rutgers University. Solid modeling of the submerged inlet is performed in ProEngineer using coordinates obtained from the computations. A STereo-Lithography (STL) file, which is a 3D faceted geometric model, is created in ProEngineer.

A comparison of the simulation results for one of the designs in Set B with the experimental measurements was performed. Compared with the baseline inlet, the distortion coefficient is improved (i.e., reduced) in both the computation and the experiment. Specifically, the DC is reduced from 0.48 (baseline inlet) to 0.08 (optimum inlet) in the simulation and from 0.27 (baseline inlet) to 0.22 (optimum inlet) in the experiment. However, there is a significant difference between the computed and experimental DC values for both the baseline and optimal inlets which is attributable to the limitations of the accuracy of the simulation (specifically, the turbulence model used in the simulations). This is a common occurrence in such complex flow configurations, and will be addressed in future work (see below).

\section{Future Work}

The second stage of our research on optimal design of subsonic submerged inlets will focus on incorporating the experiment into the design optimization using 


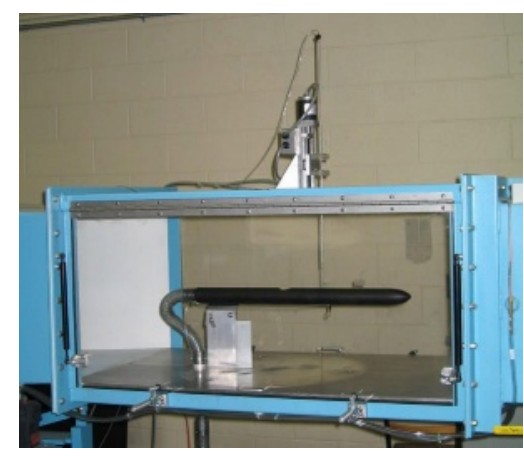

Fig. 6. Experimental facility

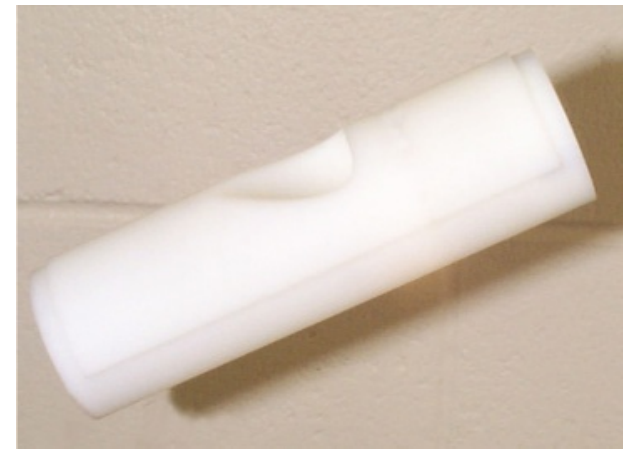

Fig. 7. Rapid prototyping model

the DDDOM software system. Two specific issues will be addressed. First, the experimental results will be used to extend the parametric range of the design optimization to non-zero angles of attack and sideslip. Evaluation of the distortion coefficient at nonzero angles of attack and sideslip is more readily (and quickly) achieved by experiment than by simulation. Thus, the experiment (rather than the simulation) will dynamically drive the optimization at nonzero angles of attack and sideslip. This demonstrates the enhancement offered by the Data Driven Design Applications System (DDDAS) concept. Second, we will incorporate the effects of uncertainty in the simulation (as defined by the results presented herein) into the DDDOM.

\section{Conclusions}

Engineering design is an example of a Dynamic Data Driven Application System wherein data from experiment and simulation can be synergized to dynamically drive the optimization process to achieve better designs in a shorter time. A Data Driven Design Optimization Methodology (DDDOM) is described which incorporates experiment and simulation in a real-time, synergistic manner. The DDDOM software system is developed using Perl and Perl/Tk. The DDDOM is applied to the multi-objective design optimization for a submerged inlet. The first stage results are presented. The Pareto Set of optimal designs at zero angle of attack and yaw are obtained, and the results compared with experiment. The uncertainty in the simulation (due to the limitations of the turbulence model) is identified. The second stage of research will extend the parametric space to nonzero angles of attack and yaw using the experimental data (rather than the simulation results) to drive the optimization.

Acknowledgments. The research is sponsored by the US National Science Foundation under grant CTS-0121058. The program managers are Drs. Frederica Darema, C. F. Chen and Michael Plesniak. 


\section{References}

1. http://www.cise.nsf.gov/eia/dddas

2. https://www.fastlane.nsf.gov/servlet/showaward?award $=0121177$.

3. https://www.fastlane.nsf.gov/servlet/showaward?award $=0121667$.

4. https://www.fastlane.nsf.gov/servlet/showaward?award $=0121263$.

5. http://www.ncsa.uiuc.edu/About/TeraGrid

6. Knight D., Elliott G., Jaluria Y., Langrana N., and Rasheed K. Automated Optimal Design Using Concurrent Integrated Experiment and Simulation. AIAA Paper No. 2002-5636, AIAA/ISSMO Symposium on Multidisciplinary Analysis and Optimization, Atlanta, GA (2002).

7. Deb, K. Multi-Objective Optimization using Evolutionary Algorithms. John Wiley \& Sons, New York (2001).

8. Knight D., Zhao H., Icoz T., and Jaluria Y. Data Driven Design Optimization Methodology. F. Darema (ed.) Dynamic Data Driven Applications Systems. Kluwer Academic Publishers, Dordrecht, The Netherlands (2004), to appear.

9. Wall L., Christiansen T., and Orwant J. Programming Perl. O'Reilly and Associates, Sebastopol, CA (2000).

10. Christiansen T., and Torkington N. Perl Cookbook. O'Reilly and Associates, Sebastopol, CA (1998).

11. Lidie S., and Walsh N. Mastering Perl/Tk. O'Reilly and Associates, Sebastopol, CA (2002).

12. E.S. Taskinoglu, V. Jovanovic, D. Knight. AIAA 2004-25 Multi-objective Design Optimization and Experimental Measurements for a Submerged Inlet. 42nd AIAA Aerospace Sciences Meeting and Exhibit, Reno, Nevada (2004).

13. E. Taskinoglu, D. Knight. Design Optimization for Subsonic Submerged Inlets. AIAA Paper 2003-1247, Jan, 2003.

14. Parametric Technology Corporation. Pro/Engineer User's Guide. (1996).

15. Program Development Corporation. GridPro/az3000 User's Guide and Reference Manual. White Plain, New York (1998).

16. Aerosoft Inc. General Aerodynamic Simulation Program User Manual. (1996). 